\title{
Caracterización física y química de Zingiber officinale en diferentes estados de maduración para su uso potencial en la elaboración de bebidas saludables \\ Physical and chemical characterization of Zingiber officinale in different maturity stages for its potential use in the elaboration of healthy beverages
}

\author{
J. Jaimez-Ordaz ${ }^{(D a}$, E. Contreras-López ${ }^{\circledR}$, F. González-Mesillas ${ }^{(D a}$, L.G. González-Olivares ${ }^{(D a}$, \\ J. Onofre-Sánchez iD b J. Ramírez-Godínez ${ }^{D}$ b* \\ ${ }^{a}$ Área Académica de Química, Instituto de Ciencias Básicas e Ingeniería, Universidad Autónoma del Estado de Hidalgo, 42184, Mineral de la Reforma, Hidalgo, \\ México. \\ ${ }^{b}$ Área Académica de Gastronomía, Instituto de Ciencias Económico Administrativas, Universidad Autónoma del Estado de Hidalgo, 42170, San Agustín Tlaxiaca, \\ Hidalgo, México.
}

\section{Resumen}

El objetivo fue caracterizar física y químicamente el jengibre (Zingiber officinale) cultivado en la Sierra Norte de Puebla, en tres estados de madurez. Las propiedades físicas determinadas fueron eje mayor, eje menor, espesor, peso, volumen y color. La composición proximal se realizó de acuerdo a la metodología de la AOAC. El contenido de minerales de determinó de acuerdo a la metodología establecida por la EPA. Las propiedades físicas cambiaron de acuerdo al estado de madurez. La humedad y fibra fueron los componentes mayoritarios en todas las muestras analizadas. En general, el contenido de $\mathrm{Ca}$, Fe y $\mathrm{Mn}$ aumentó conforme el estado de madurez del jengibre mientras que el de $\mathrm{Cu}$ y $\mathrm{P}$ disminuyó. El curcumeno, geraniol, zingibereno, bisaboleno y neral fueron identificados en el jengibre estudiado. Las propiedades que presentan los rizomas pueden ser aprovechadas en el procesamiento de productos derivados saludables.

Palabras Clave:

Jengibre, Zingiber officinale, propiedades físicas, composición química, minerales

\begin{abstract}
The objective was to characterize physically and chemically the ginger (Zingiber officinale) grown in the Sierra Norte de Puebla, in three stages of maturity. The physical properties determined were major axis, minor axis, thickness, weight, volume, and color. The proximate composition was analyzed according to the AOAC methodology. The mineral content was determined according to the methodology established by the EPA. The physical properties changed according to the state of maturity. Moisture and fiber were the major components in all the samples analyzed. In general, the content of $\mathrm{Ca}, \mathrm{Fe}$ and $\mathrm{Mn}$ increased according to the state of maturity of the ginger while that of $\mathrm{Cu}$ and $\mathrm{P}$ decreased. Curcumene, geraniol, zingiberene, bisabolene, and neral were identified in the studied ginger. The properties of rhizomes can be used in the processing of healthy derived products.
\end{abstract}

Keywords:

Ginger, Zingiber officinale, physical properties, chemical composition, minerals

\section{Introducción}

El jengibre (Zingiber officinale) es originario de las zonas tropicales del sureste asiático. Es una planta perteneciente a la familia Zingiberaceae que posee alrededor de 45 géneros y más de 1000 especies. La parte más usada de esta planta es el rizoma, el cual ha sido extensivamente utilizado desde hace miles de años como especia y medicina tradicional, principalmente en China y en la India (Shukla y Singh, 2007).
La composición del rizoma de jengibre incluye carbohidratos, vitaminas, fibra, minerales y enzimas proteolíticas entre otros componentes al igual que numerosas sustancias que además de impartirle su sabor y aroma característicos, le confieren ciertas propiedades farmacológicas y fisiológicas probadas ((Shukla y Singh, 2007; Ali et al., 2008; Shahrajabian et al, 2019).

Los componentes del jengibre consisten principalmente en hidrocarburos sesquiterpénicos tales como zingibereno, curcumeno, farneseno, bisaboleno y $\beta$-sesquifelandreno. También

\footnotetext{
*Autor para la correspondencia: juan_ramirez@uaeh.edu.mx

Correo electrónico: jjaimez@uaeh.edu.mx (Judith Jaimez-Ordaz), elizac@uaeh.edu.mx (Elizabeth Contreras-López), francisco.gon.mes@gmail.com (Francisco González Mesillas), lgonzales@uaeh.edu.mx (Luis Guillermo González-Olivares), jair_onofre6570@uaeh.edu.mx (Jair Emmanuel Onofre-Sánchez) y juan_ramirez@uaeh.edu.mx (Juan RamírezGodínez) 
están presentes en menor proporción hidrocarburos monoterpenoides como linalool, neral y geraniol (Shukla \& Singh, 2007; Shahrajabian et al, 2019). Otros componentes principales del jengibre son de tipo fenólico como los gingeroles, shogaoles y paradoles (Shukla \& Singh, 2007; Stoilova et al, 2007; Lu et al, 2014; Gümüşaya et al, 2015).

El jengibre ha sido utilizado en el tratamiento de diferentes síntomas de enfermedad como vómito, náusea, desórdenes gastrointestinales y enfermedades como tos, gripa y bronquitis (Stoilova et al, 2007; Lu et al, 2014). También resulta eficaz para tratar la artritis, reumatismo, malestares musculares e inflamación. Otros beneficios del jengibre que han sido científicamente probados incluyen efectos antimicrobianos, hipoglucémicos, analgésicos, neuroprotectores, anticancerígenos, antiinflamatorios y antioxidantes (Shahrajabian et al, 2019). Investigaciones recientes han demostrado la actividad in silico de algunos componentes del jengibre contra el coronavirus (COVID19) (Rajabopal et al. 2020) y una mejora en síntomas como fiebre, tos y cansancio en pacientes positivos a dicha enfermedad (Safa et al., 2020).

Como consecuencia de sus múltiples efectos benéficos para la salud, el jengibre se ha considerado como una materia prima alternativa en la industria alimentaria principalmente en el subsector de las bebidas saludables y/o funcionales. Por lo anterior, el objetivo del presente trabajo fue determinar las propiedades fisicoquímicas de jengibre cultivado en la Sierra Norte de Puebla, en diferentes estados de maduración, así como la identificación de los principales compuestos presentes en él con la finalidad de determinar su uso en la elaboración de bebidas saludables.

\section{Metodología}

\subsection{Obtención de las muestras}

Se analizaron rizomas de jengibre orgánico, frescos, recién cosechados en tres estados de maduración: 3,5 y 8 meses (3M, $5 \mathrm{M}$ y $8 \mathrm{M}$, respectivamente), provenientes del municipio de Xicotepec, Puebla. Los rizomas recién cosechados se transportaron en costales de malla de polietileno, posteriormente se almacenaron en bolsas de polietileno en un congelador a $-18{ }^{\circ} \mathrm{C}$ hasta su análisis.

Las muestras $3 \mathrm{M}$ y $8 \mathrm{M}$ fueron proporcionadas lavadas y limpias por parte de la empresa, mientras que la muestra $5 \mathrm{M}$ fue suministrada directamente por lo que se le realizó un lavado y cepillado dentro del laboratorio. Una vez limpios, los rizomas de los tres estados de maduración se esparcieron en una superficie plana y se seleccionaron aleatoriamente por cuarteo para la obtención de una muestra representativa de cada uno de los lotes analizados (3M, 5M, $8 \mathrm{M})$.

\subsection{Caracterización física}

Se midieron cuatro parámetros: dimensión, peso, volumen y número de ramificaciones. Para medir la dimensión (espesor, eje mayor y menor) se utilizó un vernier. El peso de los rizomas enteros se determinó con balanza granataria, clasificándose según su peso en 3 calibres: "A" grande $(\geq 300 \mathrm{~g})$, "B" mediano (200$299 \mathrm{~g})$, "C" pequeño (151-199g) según la norma del Codex Alimentarius para el jengibre (CODEX STAN 128-1999, 2005).
Para fines de esta investigación se agregó el calibre " $D$ " ( $\geq$ $150 \mathrm{~g}$ ), debido a que algunas muestras, en especial las de etapas tempranas de maduración, se encontraban fuera del establecido. El volumen se determinó mediante la inmersión de los rizomas en vasos de precipitados $(1000 \mathrm{~mL})$, donde la cantidad de agua desplazada constituyó el volumen de los rizomas. Por último, se determinó el número de ramificaciones presentes en cada una de las muestras.

\section{Color.}

El color externo de los rizomas se midió instrumentalmente, utilizando un colorímetro HunterLab determinando los parámetros CIE L* (luminosidad), a* (coordenada rojo-verde) y $\mathrm{b}^{*}$ (coordenada amarillo-azul).

\subsection{Composición química}

Se determinó utilizando los métodos oficiales de la AOAC: humedad AOAC 925.19 (2005), proteína AOAC 920.165 (1999), extracto etéreo AOAC 920.39 (2005), cenizas AOAC 941.12 (2005), fibra cruda AOAC 962.09 (1999) y carbohidratos por diferencia. El análisis de minerales $(\mathrm{Ca}, \mathrm{Cu}, \mathrm{Fe}, \mathrm{Mn}, \mathrm{P})$ se realizó por espectroscopía de emisión atómica con plasma acoplado inductivamente (ICP) de acuerdo con el método 3052, de la Agencia de Protección Ambiental (EPA: Environmental Protection Agency, 1996).

\section{Resultados y discusión}

\subsection{Caracterización física}

Tamaño y forma

Los resultados promedio del tamaño y forma (Figura 1) de las muestras de jengibre analizadas $(3 \mathrm{M}, 5 \mathrm{M}, 8 \mathrm{M})$ se muestran en la Tabla 1.

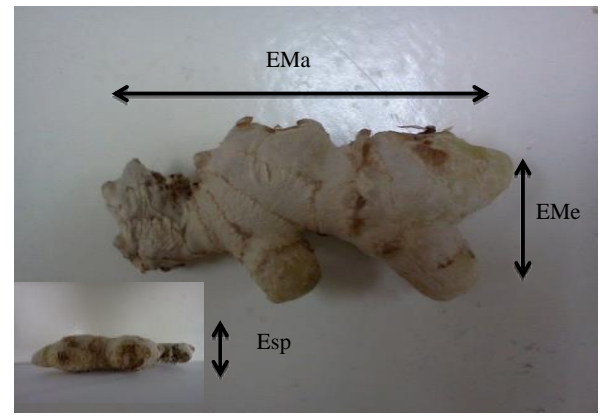

Figura 1: Medición del tamaño y forma del jengibre (Esp, espesor; Ema, eje mayor; Eme, eje menor)

Tabla 1: Tamaño promedio de las muestras de jengibre estudiadas

\begin{tabular}{cccc}
\hline Dimensiones & $3 \mathrm{M}$ & $5 \mathrm{M}$ & $8 \mathrm{M}$ \\
\hline $\begin{array}{c}\text { Eje mayor } \\
(\mathrm{cm})\end{array}$ & $6.11 \pm 0.06^{\mathrm{a}}$ & $13.45 \pm 0.1^{\mathrm{b}}$ & $13.85 \pm$ \\
& & & $0.09^{\mathrm{c}}$ \\
Eje menor & $3.08 \pm 0.08^{\mathrm{a}}$ & $6.59 \pm 0.15^{\mathrm{b}}$ & $7.48 \pm 0.12^{\mathrm{c}}$ \\
$(\mathrm{cm})$ & & & \\
Espesor $(\mathrm{cm})$ & $1.52 \pm 0.05^{\mathrm{a}}$ & $3.67 \pm 0.04^{\mathrm{c}}$ & $2.88 \pm 0.07^{\mathrm{b}}$ \\
\hline
\end{tabular}

Superíndices diferentes dentro de la misma fila, denotan diferencia significativa a un nivel de significancia del $95 \%$. Media \pm SD: desviación estándar de 3 réplicas

Akhtar, Kumar y Mannan (2013) reportaron datos de eje mayor $(4.7-10.44 \mathrm{~cm})$, eje menor $(1.58-4.6 \mathrm{~cm})$ y espesor $(1.96-4.44 \mathrm{~cm})$ para 10 muestras de germoplasmas de jengibre maduro cultivado en Bangladesh mientras que Onu y Okafor (2002) y Ajav y 
Ogundale (2014) determinaron los mismos parámetros en jengibre nigeriano (Zingiber officinale) obteniendo valores de 3-13.3 (eje mayor), $1.8-8.2 \mathrm{~cm}$ (eje menor) y $0.9-4.4 \mathrm{~cm}$ (espesor).

Comparando los resultados obtenidos en el presente trabajo, las muestras eran, en general, de tamaño similar a las muestras de jengibre cultivadas en Nigeria mientras que las muestras $3 \mathrm{M}$ y $5 \mathrm{M}$ eran más pequeñas comparadas con el jengibre de Ecuador. Únicamente las muestras $8 \mathrm{M}$ resultaron similares en tamaño y forma a los datos reportados por Torres (2011) para muestras de jengibre ecuatoriano (13.81 y $7.72 \mathrm{~cm}$, respectivamente). Las muestras $5 \mathrm{M}$ fueron similares a las reportadas por Zubair et al. (2020) $(10.85 \mathrm{~cm} ; 6.91 \mathrm{~cm}$ y 3.52 de eje mayor, eje menor y espesor, respectivamente) para muestras de jengibre var. Kochin procedente de la India. Las propiedades físicas del jengibre estudiado mostraron variabilidad lo cual es típico en biomateriales ya que distintos factores como el estado de madurez, la especie y variedad, las condiciones de cultivo y condiciones climáticas ejercen gran influencia sobre dichas características (Orellana, 2004; Ajav y Ogundale, 2014). Estas propiedades juegan un papel importante en el manejo poscosecha y en la calidad de los productos finales derivados de jengibre (Ajav y Ogundale, 2014; Zubair et al., 2020).

Peso

La Tabla 2 muestra el peso promedio de los rizomas analizados. Ninguna de las muestras analizadas alcanzó el calibre "A" establecido por la norma CODEX STAN 218-1999, que es para muestras con pesos mayores a $300 \mathrm{~g}$. Alrededor del $18 \%$ de las muestras 5M y 8M se encontraron dentro del calibre "B" (200$299 \mathrm{~g}$ ) mientras que $21 \%$ de las muestras $8 \mathrm{M}$ y sólo $8 \%$ de las muestras 5M alcanzaron el calibre "C" $(151-199 \mathrm{~g})$. Todas las muestras $3 \mathrm{M}$ y la mayoría de las muestras $5 \mathrm{M}$ y $8 \mathrm{M}$ presentaron pesos inferiores a $150 \mathrm{~g}$ lo que las coloca en el calibre " $\mathrm{D}$ ".

Tabla 2: Tamaño promedio de las muestras de jengibre estudiadas

\begin{tabular}{cccc}
\hline Muestra & $\begin{array}{c}\text { Peso del } \\
\text { lote }(\mathrm{g})\end{array}$ & $\begin{array}{c}\text { Total de } \\
\text { rizomas }\end{array}$ & $\begin{array}{c}\text { Peso } \\
\text { promedio* }\end{array}$ \\
\hline $3 \mathrm{M}$ & 1469.07 & 134 & 10.96 \\
$5 \mathrm{M}$ & 3759.47 & 32 & 117.48 \\
$8 \mathrm{M}$ & 3739.05 & 37 & 101.05 \\
\hline \multicolumn{4}{c}{ *Peso promedio por rizoma (gramos) }
\end{tabular}

Las muestras de 3 meses de maduración analizadas en este trabajo se pueden catalogar como jengibre "baby" ya que eran rizomas muy jóvenes. Es conocido que tanto el tamaño como el peso del rizoma aumenta conforme avanza el estado de madurez, el jengibre "baby" es más pequeño y suave mientras que el jengibre maduro es más grande y fibroso. Los pesos promedio de las muestras $5 \mathrm{M}$ y $8 \mathrm{M}$ fueron similares. Sin embargo; algunas muestras $8 \mathrm{M}$ alcanzaron pesos de entre 151 y $199 \mathrm{~g}$ debido al estado de maduración. La empresa que proporcionó las muestras para su análisis generalmente comercializa los rizomas al alcanzar, al menos, los 8 meses de edad. En este estado de madurez las muestras ya presentan pesos superiores a los $100 \mathrm{~g}$ (Flores, 2012).

Los resultados obtenidos son similares a los reportados en otros estudios sobre jengibre procedente de Nigeria, Ecuador y Bangladesh donde determinaron algunos parámetros físicos de las muestras, entre ellos el peso (Onu y Okafor 2002; Torres, 2011 y Akhtar, Kumar y Mannan, 2013). Al igual que en este trabajo, todas las muestras de jengibre analizadas por los autores mencionados se encontraron fuera de la clasificación por calibres establecidas por el Codex Alimentarius ya que el peso promedio mínimo determinado fue de $10.8 \mathrm{~g}$ y el máximo fue de $122.06 \mathrm{~g}$. Sin embargo, en el caso de los estudios citados previamente, no se menciona el estado de maduración del jengibre utilizado por lo que no es posible correlacionar la edad con los parámetros físicos analizados.

\section{Número de ramificaciones o brotes}

Las muestras $3 \mathrm{M}$ presentaron 2 ramificaciones en promedio mientras que los rizomas de $5 \mathrm{M}$ y $8 \mathrm{M}$ presentaron 6 . Los rizomas más jóvenes (3M) eran más uniformes debido a que sus ramificaciones eran menos pronunciadas comparados con los más maduros $(5 \mathrm{M}$ y $8 \mathrm{M})$ cuyas ramificaciones eran más alargadas y carnosas. Estos resultados concuerdan con lo reportado por Acuña y Torres (2010), quienes determinaron ramificaciones pronunciadas, alargadas y de estructura carnosa en las muestras de jengibre analizadas. Sin embargo, el número promedio de ramificaciones presentes por rizoma reportado por dichos autores fue de $3 \pm 1$.

\section{Volumen}

Los volúmenes obtenidos fueron $21 \mathrm{~cm}^{3}, 210 \mathrm{~cm}^{3}$ y $245 \mathrm{~cm}^{3}$ como valores promedio para las muestras $3 \mathrm{M}, 5 \mathrm{M}$ y $8 \mathrm{M}$, respectivamente. Estos valores son superiores a los datos (7.43$133 \mathrm{~cm}^{3}$ ) reportados por Onu y Okafor (2002) y Torres (2011) pero inferiores a los reportados por Ajav y Ogundale (2014).

La información acerca del peso, tamaño, forma, número de ramificaciones y volumen de muestras de jengibre es muy escasa en la literatura ya que no suelen medidos. Sin embargo, estos parámetros son relevantes durante el procesamiento del jengibre ya que influyen en la eficiencia del pelado y rebanado (Onu y Okafor, 2002) así como en la presentación y calidad del producto final (aceite esencial, condimento en polvo, infusión filtrante, aromatizantes, etc.) (Acuña y Torres, 2010; Ajav y Ogundale, 2014; Zubair et al., 2020).

\section{Color}

Las lecturas promedio de los parámetros $\mathrm{L}^{*}, \mathrm{a}^{*}$ y $\mathrm{b}^{*}$ del jengibre analizado se muestran en la Tabla 3.

Tabla 3: Parámetros de color medidos en las muestras de jengibre analizadas

\begin{tabular}{cccc}
\hline Parámetro & $3 \mathrm{M}$ & $5 \mathrm{M}$ & $8 \mathrm{M}$ \\
\hline $\mathrm{L}^{*}$ & $22.92 \pm 0.057^{\mathrm{a}}$ & $18.20 \pm 0.300^{\mathrm{c}}$ & $19.95 \pm 0.131^{\mathrm{b}}$ \\
$\mathrm{a}^{*}$ & $2.40 \pm 0.026^{\mathrm{c}}$ & $4.55 \pm 0.062^{\mathrm{a}}$ & $2.63 \pm 0.087^{\mathrm{b}}$ \\
$\mathrm{b}^{*}$ & $16.84 \pm 0.060^{\mathrm{a}}$ & $14.51 \pm 0.097^{\mathrm{b}}$ & $7.78 \pm 0.070^{\mathrm{c}}$ \\
\hline
\end{tabular}

Superíndices diferentes dentro de la misma fila, denotan diferencia significativa a un nivel de significación del 95\% Media \pm SD: desviación estándar de 3 réplicas

El análisis estadístico realizado indicó que existen diferencias significativas entre estos parámetros para todas las muestras, aunque a simple vista, la muestra $3 \mathrm{M}$ se percibió de un color amarillo claro brillante mientras que las muestras 5 y $8 \mathrm{M}$ presentaron un color amarillo más oscuro y opaco. Estos resultados difieren de los reportados para jengibre fresco nigeriano $\left(\mathrm{L}^{*}=59.8, \mathrm{a}^{*}=3.7 \mathrm{y} \mathrm{b}=16.9\right)($ Okafor y Okafor, 2007) y a lo reportado por Osae et al. (2019) $\left(\mathrm{L}^{*}=50.33, \mathrm{a}^{*}=1.82\right.$, $\left.b^{*}=35.72\right)$ para jengibre fresco originario de China. 
El color del jengibre fresco varía del dorado al café pálido dependiendo de su origen. Por ejemplo, el jengibre jamaicano es dorado brillante, el africano es de color más oscuro y el chino es de color más blanco o pálido. Este parámetro también depende de las condiciones de cosecha, almacenamiento y procesado (Uhl, 2000).

\subsection{Caracterización química}

Los resultados obtenidos de la caracterización química de las muestras de jengibre de 3M, $5 \mathrm{M}$ y $8 \mathrm{M}$ se presentan en la Tabla 4. Se observaron diferencias significativas en cuanto al contenido de humedad, cenizas, fibra y carbohidratos. Las muestras $3 \mathrm{M}$ y $8 \mathrm{M}$ presentaron contenidos similares de grasa y las muestras $5 \mathrm{M}$ y $8 \mathrm{M}$, de proteína. Se observó que el estado de madurez influyó de manera significativa sobre el porcentaje de cenizas, siendo mayor en el estado más joven puesto que al inicio de su crecimiento necesita de estos elementos para un adecuado desarrollo. Respecto al contenido de fibra, este aumenta conforme avanza el estado de madurez. Los rizomas maduros son fibrosos y secos, en comparación con los rizomas jóvenes que son jugosos y carnosos (Martínez et al., 2013).

El contenido de humedad de las muestras analizadas se encontró entre 85 y $89 \%$. Estos valores concuerdan con los datos reportados en la literatura, los cuales se encuentran en un intervalo de 71 a 95\% (Mao et al., 2019; Osae et al., 2019; Shukla et al., 2019; Zubair et al., 2020) y coinciden con el contenido de humedad reportado para las principales variedades comerciales de jengibre en el mundo (Govindarajan y Connell, 1983). Los resultados del contenido de fibra de los rizomas analizados (4 a $10 \%$ ) son similares a los presentados por Kumar et al. (2013) y por Shukla et al. (2019) pero inferiores al intervalo publicado (8$20 \%$ ) para la mayoría de las muestras de jengibre comerciales en el mundo (Govindarajan y Connell, 1983). En cuanto a los parámetros de grasa, cenizas, proteína y carbohidratos los valores obtenidos en esta investigación son inferiores a los reportados en la literatura (Kumar et al., 2013; Shukla et al., 2019 y Zubair et al., 2020).

Tabla 4: Composición proximal porcentual y cuantificación de minerales presentes (mg de mineral/100g) en el jengibre

\begin{tabular}{crrr}
\hline Parámetro & \multicolumn{1}{c}{$3 \mathrm{M}$} & \multicolumn{1}{c}{$5 \mathrm{M}$} & \multicolumn{1}{c}{$8 \mathrm{M}$} \\
\hline Humedad & $88.98 \pm 0.85^{\mathrm{a}}$ & $86.95 \pm 1.12^{\mathrm{b}}$ & $85.28 \pm 0.41^{\mathrm{c}}$ \\
Cenizas & $1.19 \pm 0.02^{\mathrm{a}}$ & $0.63 \pm 0.06^{\mathrm{c}}$ & $0.74 \pm 0.02^{\mathrm{b}}$ \\
Grasa & $0.12 \pm 0.01^{\mathrm{b}}$ & $0.24 \pm 0.02^{\mathrm{a}}$ & $0.14 \pm 0.01^{\mathrm{b}}$ \\
Proteína & $1.84 \pm 0.14^{\mathrm{a}}$ & $1.33 \pm 0.14^{\mathrm{b}}$ & $1.37 \pm 0.12^{\mathrm{b}}$ \\
Fibra & $4.26 \pm 0.42^{\mathrm{c}}$ & $8.91 \pm 0.81^{\mathrm{b}}$ & $9.96 \pm 0.07^{\mathrm{a}}$ \\
Carbohidratos & $3.61 \pm 0.08^{\mathrm{a}}$ & $1.94 \pm 0.25^{\mathrm{c}}$ & $2.51 \pm 0.33^{\mathrm{b}}$ \\
Ca & $153.71 \pm 3.23^{\mathrm{b}}$ & $220.34 \pm 26.09^{\mathrm{a}}$ & $218.24 \pm 12.46^{\mathrm{a}}$ \\
$\mathrm{Cu}$ & $39.78 \pm 4.58^{\mathrm{a}}$ & $37.30 \pm 9.24^{\mathrm{a}}$ & $0.20 \pm 0.01^{\mathrm{b}}$ \\
$\mathrm{Fe}$ & $24.14 \pm 3.10^{\mathrm{c}}$ & $38.41 \pm 2.71^{\mathrm{b}}$ & $96.18 \pm 12.74^{\mathrm{a}}$ \\
$\mathrm{P}$ & $7.34 \pm 0.18^{\mathrm{a}}$ & $3.88 \pm 0.62^{\mathrm{b}}$ & $0.01 \pm 0.002^{\mathrm{c}}$ \\
$\mathrm{Mn}$ & $18.46 \pm 0.42^{\mathrm{b}}$ & $17.89 \pm 1.49^{\mathrm{b}}$ & $176.05 \pm 48.89^{\mathrm{a}}$ \\
\hline
\end{tabular}

Superíndices diferentes dentro de la misma fila, denotan diferencia significativa a un nivel de significación del 95\% Media \pm SD: desviación estándar de 3 réplicas

Respecto al contenido de minerales, se observó que el Ca, Fe y Mn aumentaron conforme el estado de madurez del jengibre mientras que el $\mathrm{Cu}$ y $\mathrm{P}$ disminuyeron (Tabla 4). En el presente estudio se observó que la concentración de Fe y Mn aumentaron significativamente después de los 5 meses de maduración de los rizomas de jengibre analizados.

Comparando los resultados del contenido de minerales del jengibre analizado con lo reportado por diferentes autores, se observa que difieren con lo encontrado por Famurewa et al. (2011) y Otunola et al. (2010); ya que el contenido de hierro y zinc son inferiores a los determinados en el presente trabajo. En cuanto al contenido de $\mathrm{Ca}, \mathrm{Cu}, \mathrm{Fe}$ y Mn obtenido por Shirin y Prakash (2010) y Tanveer et al. (2014), es superior al encontrado en las diferentes etapas de maduración del jengibre estudiado. De igual forma, Latona et al. (2012) reportaron valores superiores de hierro. Sin embargo, el contenido de $\mathrm{P}$ es inferior a lo reportado por estos autores. En contraste, el contenido de minerales del jengibre en los diferentes estados de maduración fue superior al determinado por Ogbuewu et al. (2014), Shukla et al. (2019) y por Zubair et al., (2020), a excepción del P cuyo contenido fue superior $(10-42$ y $30.93 \mathrm{mg} / 100 \mathrm{~g})$ en los últimos estudios mencionados. Cabe destacar que los autores citados no indican el estado de maduración de los rizomas analizados, aunque generalmente, el jengibre se cosecha entre 6 y 12 meses posteriores a la siembra (Ajav y Ogundale, 2014).

Las variaciones observadas en la composición química tanto del jengibre analizado como de los resultados reportados en la literatura podrían atribuirse a que los componentes químicos del jengibre, así como la calidad y cantidad de los constituyentes biológicamente activos presentes en él pueden variar considerablemente dependiendo de factores como la especie, madurez, ubicación del cultivo, condiciones agro-climáticas, tratamiento postcosecha o de si el producto es fresco, seco o procesado (Singletary, 2010; Shahrajabian et al, 2019). Cabe mencionar que, en este estudio, las muestras se analizaron en estado fresco y todas provenían del mismo lugar de origen, aunque de parcelas diferentes por lo que pudo haber variación en la composición del suelo.

Se ha reportado que una fuente de variación en el contenido de minerales es la fertilización de los cultivos, aunque en este caso, las muestras de jengibre utilizadas provienen de parcelas libres de fertilizantes dado que la empresa que proporcionó las muestras para su estudio (Productores Orgánicos de Black Berry de la Sierra Norte de Puebla S.C. de R.L.) se dedica a la producción, empaque y comercialización de jengibre orgánico.

\section{Conclusiones}

Las propiedades físicas y químicas del jengibre están relacionadas con el estado de maduración y con factores asociados con su origen y con las condiciones de cultivo.

Es importante considerar el estado de madurez del jengibre a utilizar para la elaboración de diversos productos derivados de este rizoma ya que la composición química varía con la maduración y algunos componentes pueden modificar atributos sensoriales de los productos finales, particularmente el color, sabor y olor.

El desarrollo de una metodología para la elaboración de una bebida saludable utilizando jengibre como ingrediente es viable debido a las propiedades determinadas en los rizomas ya que algunos compuestos presentes reportados en literatura como el zingibereno, bisaboleno y geraniol poseen propiedades antioxidantes. Además, el jengibre es una fuente rica de minerales 
y fibra. Lo anterior permite que pueden ser aprovechadas en el procesamiento de productos derivados de jengibre.

\section{Agradecimientos}

Los autores agradecen a Productores Orgánicos de Black Berry de la Sierra Norte de Puebla S.C. de R.L. la donación de las muestras de jengibre y al financiamiento por parte de PRODEP por el apoyo a la incorporación de nuevos PTC.

\section{Referencias}

Acuña, O., Torres, A. (2010). Aprovechamiento de las propiedades funcionales del jengibre (Zingiber officinale $\mathrm{R}$.) en la elaboración de condimento en polvo, infusión filtrante y aromatizante para quema directa. Revista Politécnica 29(1), 6069.

Ajav, A., Ogunlade, C. A. (2014). Physical Properties of Ginger (Zingiber Officinale). Global Journal of Science Frontier $\begin{array}{lll}\text { Research } & 14(8), & 1-9 .\end{array}$ https://journalofscience.org/index.php/GJSFR/article/view/140 $1 / 1263$

Akhtar, A., Dash, K. P., Mannan, A. (2013). Physico-Chemical Characterization and Product Development from Ginger (Zingiber officinalis) Germplasm Available in South Western Region of Bangladesh. Journal of Agriculture and Veterinary Science 5(6), 53-62.

Ali, B.H., Blunden, G., Tamira, O.M., Nemmar, A. (2008). Some phytochemical, pharmacological and toxicological properties of ginger (Zingiber officinale Roscoe): A review of recent research. Food and Chemical Toxicology 46, 409-420. DOI: https://doi.org/10.1016/j.fct.2007.09.085

AOAC. (2005). Official Methods of Analysis. Association of Official Analytical Chemist. (18th ed). Washington:USA

CODEX STAN 128-1999. (2005). Norma del Codex para el jengibre. Food and Agriculture Organization of the United Nations. Rome:Italy

Environmental Protection Agency (EPA). (1996). Method 3052. Microwave assisted acid digestion of siliceous and organically based matrices. Washington:USA

Famurewa, A.V., Emuekele, P.O., Jaiyeoba. K.F. (2011). Effect of drying and size reduction on the chemical and volatile oil contents of ginger (Zingiber officinale). Journal of Medicinal Plants Research 5(14), 2941-2944.

Flores, K. (2012). Baby ginger: Easy and extraordinary niche crop. Rodale Institute. Pennsylvania:USA

Govindarajan, V. S., Connell, D. W. (1983). Ginger - chemistry, technology, and quality evaluation: Part 1. C R C Critical Reviews in Food Science and Nutrition 17(1), 1-96. DOI: https://doi.org/10.1080/10408398209527343

Gümüşaya, Ö.A., Borazanb, A.A., Ercalc, N., Demirkold, O. (2015). Drying effects on the antioxidant properties of tomatoes and ginger. Food Chemistry 173, 156-162. DOI: https://doi.org/10.1016/j.foodchem.2014.09.162

Kumar, S., Saxena, K., Singh, U. N., Saxena, R. (2013). Antiinflammatory action of ginger: A critical review in anemia of inflammation and its future aspects. International Journal of Herbal Medicine 1 (4), 16-20.

Latona, D. F., Oyeleke, G. O., Olayiwola, O. A. (2012). Chemical Analysis of Ginger Root. Journal of Applied Chemistry 1(1), 4749.

Lu, D.L., Li, X.Z., Dai, F., Kang, Y.F., Li, Y., Ma, M,M,, Ren, X.R., Du, G.W., Jin, X.L., Zhou, B. (2014). Influence of side chain structure changes on antioxidant potency of the [6]- gingerol related compounds. Food Chemistry 165, 191-197. DOI: https://doi.org/10.1016/j.foodchem.2014.05.077

Mao, Qian-Qian; Xu, Xiao-Yu; Cao, Shi-Yu; Gan, Ren-You; Corke, Harold; Beta, Trust; Li, Hua-Bin. (2019). Bioactive Compounds and Bioactivities of Ginger (Zingiber officinale $\begin{array}{llll}\text { Roscoe). } & \text { Foods } & 8, & \text { (6), }\end{array}$ https://doi.org/10.3390/foods8060185

Martínez, A. O. L., Ardila, C. M., García, B. Y., Restrepo, C. S. (2013). Identificación y selección de descriptores de jengibre (Zingiber officinalis) con jueces entrenados para establecer un perfil sensorial por aproximación multidimensional según NTC 3932; Universidad de Antioquia. Medellín-Colombia.

Ogbuewu, I. P., Jiwuba, P. D., Ezeokeke, C. T., Uchegbul, M. C., Okoli, I. C., Iloeje, M. U. (2014). Evaluation of Phytochemical and Nutritional Composition of Ginger Rhizome Powder. International Journal of Agriculture and Rural Development 17, 1663-1670.

Okafor, G. I., Okafor, J. N. (2007). Effects of pricking, sun-drying and sieving on Ginger (Zingiber officinale Roscoe) colour and powder. Nigerian Food Journal 25, 155-160. DOI: 10.4314/nifoj.v25i1.33664

Onu, L. I., Okafor, G. I. (2002). Effect of physical and chemical factor variations on the efficiency of mechanical slicing of Nigerian ginger (Zingiber Officinale Rose). Journal of Food Engineering 56, 43-47. DOI: https://doi.org/10.1016/S02608774(02)00146-2

Orellana, A. R. (2004). Evaluacion del efecto de dos fuentes de fertilizantes en el rendimiento de jengibre (Zingiber officinale R.), en la finca bulbuxya, San Miguel Panan, Suchitepequez [Tesis de licenciatura]. Universidad de San Carlos de Guatemala, Guatemala.

Osae, R., Essilfie, G., Alolga, R. N., Bonah, E., Ma, H., Zhou, C. (2019). Drying of ginger slices-Evaluation of quality attributes, energy consumption, and kinetics study. Journal of Food Process Engineering 2019, 1-14. DOI: https://doi.org/10.1111/jfpe.13348

Otunola, G., Oloyede, B. Oladiji, T. Afolayan, A. (2010). Comparative analysis of the chemical composition of three spices - Allium sativum L. Zingiber officinale Rosc. and Capsicum frutescens L. commonly consumed in Nigeria. African Journal of Biotechnology 9 (41), 6927- 6931. DOI: 10.5897/AJB10.183

Rajagopal, K., Byran, G., Jupudi, S., Vadivelan, R. (2020). Activity of phytochemical constituents of black pepper, ginger, and garlic against coronavirus (COVID-19): An in silico approach. International Journal of Health \& Allied Sciences 9 (5), 43-50. DOI: 10.4103/ijhas.IJHAS_55_20

Safa, O., Hassaniazad, M., Farashahinejad, M. Davoodian, P., Dadvand, H., Hassanipour, S., Fathalipour, M. (2020). Effects of Ginger on clinical manifestations and paraclinical features of patients with Severe Acute Respiratory Syndrome due to COVID-19: A structured summary of a study protocol for a randomized controlled trial. Trials 21, 841. DOI: https://doi.org/10.1186/s13063-020-04765-6

Shahrajabian, M. H., Sun, W., Cheng, Q. (2019). Clinical aspects and health benefits of ginger (Zingiber officinale) in both traditional Chinese medicine and modern industry. Acta Agriculturae Scandinavica, Section B - Soil \& Plant Science 69, 546-556. DOI: https://doi.org/10.1080/09064710.2019.1606930

Shirin, A., Prakash, J. (2010). Chemical composition and antioxidant properties of ginger root (Zingiber officinale). Journal of Medicinal Plants Research 4 (24), 2674-2679. DOI: 10.5897/JMPR09.464 
Shukla, A., Goud, V. V., Das, C. (2019). Antioxidant potential and nutritional compositions of selected ginger varieties found in Northeast India. Industrial Crops \& Products 128, 167-176. DOI: https://doi.org/10.1016/j.indcrop.2018.10.086

Shukla, Y., Singh, M. (2007). Cancer preventive properties of ginger: A brief review. Food and Chemical Toxicology 45(5), 683-690. DOI: https://doi.org/10.1016/j.fct.2006.11.002

Singletary, K. Ginger. An Overview of Health Benefits. Nutrition Today 45 (4), 171-183. DOI: 10.1097/NT.0b013e3181ed3543

Stoilova, I., Krastanov, A., Stoyanova, A., Denev, P., Gargova, S. (2007). Antioxidant activity of a ginger extracts (Zingiber officinale). Food Chemistry 102, 764-770. DOI: https://doi.org/10.1016/j.foodchem.2006.06.023
Tanveer, S., Shahzad, A., Ahmed, W. (2014). Compositional and mineral profiling of Zingiber Officinale. Pakistan Journal of Food Sciences 24(1), 21-26.

Torres, M. A. (2011). Aprovechamiento de las propiedades funcionales del jengibre (Zingiber officinale) en la elaboración de un condimento en polvo, infusión filtrante y aromatizante para quema directa [Tesis de ingeniería]. Escuela Politécnica Nacional. Ecuador.

Uhl, R. S. (2000). Handbook of spices seasonings flavourings. Boca Raton, Fl: CRC Press.

Zubair, S., Sawate, A. R., Kshirsagar, R. B., Agarkar, B. S., Patil, B. M. (2020). Studies on impact of different processing methods on phyto-chemical and antioxidant activity of dried ginger (Zingiber officinale L.) rhizome. Journal of Pharmacognosy and Phytochemistry 9(4), 3153-3158. 